TRANSACTIONS OF THE

AMERICAN MATHEMATICAL SOCIETY

Volume 356, Number 8, Pages 2989-3004

S 0002-9947(04)03587-1

Article electronically published on March 23, 2004

\title{
EIGENFUNCTIONS OF THE LAPLACIAN ACTING ON DEGREE ZERO BUNDLES OVER SPECIAL RIEMANN SURFACES
}

\author{
MARCO MATONE
}

\begin{abstract}
We find an infinite set of eigenfunctions for the Laplacian with respect to a flat metric with conical singularities and acting on degree zero bundles over special Riemann surfaces of genus greater than one. These special surfaces correspond to Riemann period matrices satisfying a set of equations which lead to a number theoretical problem. It turns out that these surfaces precisely correspond to branched covering of the torus. This reflects in a Jacobian with a particular kind of complex multiplication.
\end{abstract}

\section{INTRODUCTION}

The problem of constructing a set of eigenfunctions of the Laplacian acting on degree zero bundles over Riemann surfaces was considered in 11. The corresponding metric is given by the modulo square of a particular holomorphic one-differential $\omega_{n, m}$. It was also shown in [1] that eigenvalues with a nontrivial dependence on the complex structure may be obtained as solutions of the equation

$$
\omega_{n^{\prime}, m^{\prime}}=c \omega_{n, m}
$$

This equation is equivalent to

$$
m_{j}^{\prime}-\sum_{k=1}^{h} \Omega_{j k} n_{k}^{\prime}=\bar{c}\left(m_{j}-\sum_{k=1}^{h} \Omega_{j k} n_{k}\right), \quad j=1, \ldots, h,
$$

where $m_{j}, n_{j}, m_{j}^{\prime}, n_{j}^{\prime}$ are integers. In this paper we find a set of solutions of such an equation. The general problem involved in (1.1) concerns the properties of the Riemann period matrix and its number theoretical structure. We will call special Riemann surfaces those with a Riemann period matrix satisfying (1.2).

\section{Primitive Differentials AND SCALAR PRODUCTS}

In this section, after fixing the notation, we introduce an infinite set of holomorphic one-differentials (we shall call them primitive differentials) which can be considered as the building blocks for our investigation. These differentials have been previously introduced in [1]. Next, we consider scalar products defined in terms of monodromy factors associated to the primitive differentials. By Riemann bilinear relations we generalize a result in [1] obtaining a relation between scalar product, monodromy factors and surface integrals. These aspects are reminiscent of

Received by the editors February 1, 2002.

2000 Mathematics Subject Classification. Primary 14H55; Secondary 11F72.

(C)2004 American Mathematical Society 
the well-known relations between area, Fuchsian dilatations and Laplacian spectra which arise for example in the framework of the Selberg trace formula.

2.1. Notation and definitions. Let $\Sigma$ be a compact Riemann surface of genus $h \geq 1$ and let $\alpha_{1}, \ldots, \alpha_{h}, \beta_{1}, \ldots, \beta_{h}$ be a symplectic basis of the first homology group $H_{1}(\Sigma, \mathbb{Z})$, that is, with intersection matrix

$$
\left(\begin{array}{ll}
\alpha \cdot \alpha & \alpha \cdot \beta \\
\beta \cdot \alpha & \beta \cdot \beta
\end{array}\right)=\left(\begin{array}{cc}
0 & \mathbb{I} \\
-\mathbb{I} & 0
\end{array}\right)
$$

where $\mathbb{I}$ is the $h \times h$ identity matrix. Let $\omega_{1}, \ldots, \omega_{h}$ be the basis of the $\mathbb{C}$ vector space of holomorphic one-differentials with the standard normalization

$$
\oint_{\alpha_{j}} \omega_{k}=\delta_{j k}
$$

The Riemann period matrix is defined by

$$
\Omega_{j k} \doteq \oint_{\beta_{j}} \omega_{k}
$$

By means of the Riemann bilinear relations [2]

$$
\int_{\Sigma} \omega_{j} \wedge \bar{\omega}_{k}=\sum_{l=1}^{h}\left(\oint_{\alpha_{l}} \omega_{j} \oint_{\beta_{l}} \bar{\omega}_{k}-\oint_{\alpha_{l}} \bar{\omega}_{k} \oint_{\beta_{l}} \omega_{j}\right)
$$

it follows that $\Omega_{i j}=\Omega_{j i}$ and $\operatorname{det} \Omega^{(2)}>0$, where $\Omega_{k j}^{(1)} \doteq \Re \Omega_{k j}$ and $\Omega_{k j}^{(2)} \doteq \Im \Omega_{k j}$. We shall denote elements in $H_{1}(\Sigma, \mathbb{Z})$ by

$$
\gamma_{p, q} \doteq p \cdot \alpha+q \cdot \beta, \quad(q, p) \in \mathbb{Z}^{2 h}
$$

in particular

$$
\oint_{\gamma_{p, q}} \omega \doteq \sum_{k=1}^{h}\left(p_{k} \oint_{\alpha_{k}} \omega+q_{k} \oint_{\beta_{k}} \omega\right)
$$

and

$$
\int_{z_{0}}^{z+\gamma_{p, q}} \omega \doteq \int_{z_{0}}^{z} \omega+\oint_{\gamma_{p, q}} \omega
$$

where $z_{0} \in \Sigma, z \in \Sigma$ and $\omega$ is an arbitrary meromorphic one-differential.

2.2. Primitive differentials. Let us consider the set $\mathcal{K}(\Sigma) \subset H^{1}(\Sigma)$ of all nonzero real, harmonic one-forms on $\Sigma$ which are integral, i.e. such that

$$
\oint_{\gamma} \alpha \in \mathbb{Z}, \quad \gamma \in H_{1}(\Sigma, \mathbb{Z})
$$

Note that $\mathcal{K}(\Sigma)$ is a lattice in the $2 h$ real vector space $H^{1}(\Sigma)$. Let us consider the set of holomorphic one-differentials

$$
\mathcal{H}(\Sigma) \doteq\left\{\omega=\pi i\left(\alpha+i^{\star} \alpha\right) \mid \alpha \in \mathcal{K}(\Sigma)\right\}
$$

where ${ }^{\star}$ denotes the conjugation operator whose action on a one-form $\eta=u(z) d z+$ $v(z) d \bar{z}$ is ${ }^{\star} \eta=-i u(z) d z+i v(z) d \bar{z}$. Note that $\alpha=\Im \omega / \pi$ and ${ }^{\star} \alpha=-i \Re \omega / \pi$. 
The elements of $\mathcal{H}(\Sigma)$ correspond to the following primitive differentials [1]:

$$
\omega_{n, m} \doteq \sum_{k=1}^{h} c_{n, m ; k} \omega_{k},
$$

where

$$
c_{n, m ; k}=\pi \sum_{j=1}^{h}\left(m_{j}-\sum_{l=1}^{h} n_{l} \bar{\Omega}_{l j}\right)\left(\Omega^{(2)^{-1}}\right)_{j k}, \quad(n, m) \in \mathbb{Z}^{2 h},
$$

$k=1, \ldots, h$. Let us set

$$
f_{n, m}(z) \doteq e^{\int_{z_{0}}^{z} \omega_{n, m}}
$$

where $z_{0}$ is fixed on $\Sigma$ and $z \in \Sigma$. Note that the monodromy of $f_{n, m}$ takes real values, that is,

$$
(n, m \mid q, p) \in \mathbb{R}, \quad(q, p) \in \mathbb{Z}^{2 h},
$$

where

$$
(n, m \mid q, p) \doteq e^{\oint_{\gamma_{p, q}} \omega_{n, m}}=\frac{f_{n, m}\left(z+\gamma_{p, q}\right)}{f_{n, m}(z)}=\exp \left[\sum_{j=1}^{h}\left(p_{j}+\sum_{k=1}^{h} q_{k} \Omega_{k j}\right) c_{n, m ; j}\right] .
$$

For later use we define the coefficients

$$
D_{k j}^{n m} \doteq m_{k} \delta_{k j}-n_{k} \bar{\Omega}_{k j},
$$

so that

$$
c_{n, m ; k}=\pi \sum_{j, l=1}^{h} D_{j l}^{n m}\left(\Omega^{(2)^{-1}}\right)_{l k} .
$$

2.3. Monodromy and scalar products. Let us define the scalar product

$$
\langle\langle n, m \mid q, p\rangle\rangle \doteq \oint_{\gamma_{p, q}} \omega_{n, m}, \quad(n, m ; q, p) \in \mathbb{Z}^{4 h} .
$$

By (2.11) we have

$$
\langle\langle n, m \mid q, p\rangle\rangle=\pi \sum_{j, k=1}^{h}\left(p_{j}+\sum_{i=1}^{h} q_{i} \Omega_{i j}\right)\left(\Omega^{(2)^{-1}}\right)_{j k}\left(m_{k}-\sum_{l=1}^{h} \bar{\Omega}_{k l} n_{l}\right),
$$

which has the properties

$$
\overline{\langle\langle n, m \mid q, p\rangle\rangle}=\langle\langle-q, p \mid-n, m\rangle\rangle=\langle\langle n, m \mid q, p\rangle\rangle-2 i \pi(p \cdot n+q \cdot m),
$$

so that

$$
\Im\langle\langle n, m \mid q, p\rangle\rangle=\Im\langle\langle m, n \mid p, q\rangle\rangle .
$$

Furthermore

$$
\langle\langle n, m \mid q, p\rangle\rangle=\frac{1}{2 \pi i} \sum_{j=1}^{h}(\langle\langle n, m \mid \hat{\jmath}, 0\rangle\rangle\langle\langle 0, \hat{\jmath} \mid q, p\rangle\rangle+\langle\langle n, m \mid 0, \hat{\jmath}\rangle\rangle\langle\langle\hat{\jmath}, 0 \mid q, p\rangle\rangle),
$$


where $\hat{\jmath} \doteq\left(\delta_{1 j}, \delta_{2 j}, \ldots, \delta_{h j}\right)$. By (2.13) and (2.19) it follows that

$$
(n, m \mid q, p)=e^{\langle\langle n, m \mid q, p\rangle\rangle}=e^{\overline{\langle\langle n, m \mid q, p\rangle\rangle}}=(-q, p \mid-n, m) .
$$

Note that by (2.19) the scalar product

$$
\langle n, m \mid q, p\rangle \doteq \Re \oint_{\gamma_{p,-q}} \omega_{n, m}=\Re\langle\langle n, m \mid-q, p\rangle\rangle=-\Re\langle\langle n, m \mid q,-p\rangle\rangle
$$

has the symmetry property

$$
\langle n, m \mid q, p\rangle=\langle q, p \mid n, m\rangle
$$

and

$$
\langle n, m \mid q, p\rangle=\langle\langle n, m \mid-q, p\rangle\rangle-i \pi(p \cdot n-q \cdot m) .
$$

Furthermore, by (2.18) and (2.19)

$$
\langle n, m \mid q, p\rangle=\pi \sum_{j, k=1}^{h}\left[\left(p_{j}-\sum_{i=1}^{h} q_{i} \Omega_{i j}^{(1)}\right)\left(\Omega^{(2)^{-1}}\right)_{j k}\left(m_{k}-\sum_{l=1}^{h} \Omega_{k l}^{(1)} n_{l}\right)+q_{j} \Omega_{j k}^{(2)} n_{k}\right] .
$$

Positivity of $\Omega^{(2)}$ implies that

$$
\langle n, m \mid n, m\rangle \geq 0,
$$

and $\langle n, m \mid n, m\rangle=0$ iff $|n, m\rangle=|0,0\rangle$. Observe that by (2.25) it follows that

$$
\langle n, m \mid n, m\rangle=\langle\langle n, m \mid-n, m\rangle\rangle .
$$

$\langle n, m \mid q, p\rangle$ can be expressed in terms of the coefficients $c_{n, m ; k}$ only:

$$
\begin{aligned}
\langle n, m \mid q, p\rangle & =\pi^{-1} \sum_{j, k=1}^{h}\left(a_{n, m ; j} \Omega_{j k}^{(2)} a_{q, p ; k}+b_{q, p ; j} \Omega_{j k}^{(2)} b_{n, m ; k}\right) \\
& =\pi^{-1} \sum_{j, k=1}^{h}\left(\langle n, m \mid 0, \hat{\jmath}\rangle \Omega_{j k}^{(2)}\langle 0, \hat{k} \mid q, p\rangle+q_{j} \Omega_{j k}^{(2)} n_{k}\right),
\end{aligned}
$$

where $a_{n, m ; k} \doteq \Re c_{n, m ; k}$ and $b_{n, m ; k} \doteq \Im c_{n, m ; k}$, that is,

$$
a_{n, m ; k}=\pi \sum_{j=1}^{h}\left(m_{j}-\sum_{l=1}^{h} n_{l} \Omega_{l j}^{(1)}\right)\left(\Omega^{(2)^{-1}}\right)_{j k}, \quad b_{n, m ; k}=\pi n_{k},
$$

$k=1, \ldots, h,(n, m) \in \mathbb{Z}^{2 h}$. Finally, we note that in deriving (2.29) we used the relation $\omega_{n, m}=\sum_{k=1}^{h}\left(\oint_{\alpha_{k}} \omega_{n, m}\right) \omega_{k}$, that is,

$$
c_{n, m ; k}=\oint_{\alpha_{k}} \omega_{n, m}=\langle\langle n, m \mid 0, \hat{k}\rangle\rangle, \quad a_{n, m ; k}=\langle n, m \mid 0, \hat{k}\rangle .
$$


2.4. Duality, surface integrals and monodromy. Let us set

$$
\rho_{n, m}^{(1)} \doteq \Re \sum_{k=1}^{h} d_{n, m ; k}^{(1)} \omega_{k} .
$$

We consider the problem of finding the structure of the coefficients $d_{n, m ; k}^{(1)}$ such that

$$
\oint_{\gamma_{p, q}} \rho_{n, m}^{(1)}=\oint_{\gamma_{m, n}} \rho_{q, p}^{(1)}
$$

$(n, m ; q, p) \in \mathbb{Z}^{4 h}$. This equation implies that

$$
d_{n, m ; k}^{(1)}=\sum_{j=1}^{h}\left(m_{j}+\sum_{l=1}^{h} n_{l} \Omega_{l j}^{(1)}\right) E_{j k}-\sum_{j=1}^{h}\left(i m_{j}+\sum_{l=1}^{h} n_{l} \Omega_{l j}^{(2)}\right) F_{j k}+i \sum_{l=1}^{h} n_{l} G_{l k},
$$

with $E_{i j}, F_{i j}, G_{i j}$ real symmetric matrices not depending on $(n, m)$. Note that the condition $\overline{d_{n, m ; k}^{(1)}}=d_{n, m ; k}^{(1)}-2 \pi i n_{k}$ is equivalent to $F_{j k}=0, G_{j k}=\pi \delta_{j k}$. We now set

$$
\rho_{n, m}^{(2)} \doteq \Re \sum_{k=1}^{h} d_{n, m ; k}^{(2)} \omega_{k}
$$

and consider the condition

$$
\oint_{\gamma_{p,-q}} \rho_{n, m}^{(2)}=\oint_{\gamma_{m,-n}} \rho_{q, p}^{(2)}
$$

That is,

$$
\begin{aligned}
d_{n, m ; k}^{(2)} & =\sum_{j=1}^{h}\left(m_{j}-\sum_{l=1}^{h} n_{l} \Omega_{l j}^{(1)}\right) E_{j k}+\sum_{j=1}^{h}\left(i m_{j}+\sum_{l=1}^{h} n_{l} \Omega_{l j}^{(2)}\right) F_{j k}+i \sum_{l=1}^{h} n_{l} G_{l k} \\
& =\overline{d_{-n, m ; k}^{(1)}} .
\end{aligned}
$$

In this case the conditions

$$
\overline{d_{n, m ; k}^{(2)}}=d_{n, m ; k}^{(2)}-2 \pi i n_{k}, \quad E_{j k}=\pi\left(\Omega^{(2)^{-1}}\right)_{j k},
$$

give $d_{n, m ; k}^{(2)}=c_{n, m ; k}$, that is,

$$
\rho_{n, m}^{(2)}=\Re \omega_{n, m} .
$$

Thus $c_{n, m ; k}$ can be fixed by imposing either the singlevaluedness of $\exp \Im \int^{z} \omega_{n, m}$ (2.13), or the duality condition (2.36). The same can be said of (2.24) satisfied by $\Re \omega_{n, m}$, together with (2.38).

We will see that positivity and symmetry of $\langle\cdot \mid \cdot\rangle$ are at the basis of the fact that the monodromy of $f_{n, m}$ under a shift of $z$ around $\gamma_{m,-n}$, is proportional to the area of the metric $\left|\omega_{n, m}\right|^{2}$. This metric defines a Laplacian of which $\left(f_{n, m} / \bar{f}_{n, m}\right)^{k}, k \in \mathbb{Z}$, are eigenfunctions. These aspects are reminiscent of the well-known relationships between hyperbolic dilatations and eigenvalues of the Poincaré Laplacian. 
By the Riemann bilinear relations we have

$$
\begin{aligned}
\int_{\Sigma} \omega_{n, m} \wedge \bar{\omega}_{q, p} & =\sum_{j=1}^{h}(\langle\langle n, m \mid 0, \hat{\jmath}\rangle\rangle \overline{\langle\langle q, p \mid \hat{\jmath}, 0\rangle\rangle}-\overline{\langle\langle q, p \mid 0, \hat{\jmath}\rangle\rangle}\langle\langle n, m \mid \hat{\jmath}, 0\rangle\rangle) \\
& =\sum_{j=1}^{h}(\langle\langle n, m \mid 0, \hat{\jmath}\rangle\rangle\langle\langle-\hat{\jmath}, 0 \mid-q, p\rangle\rangle-\langle\langle n, m \mid \hat{\jmath}, 0\rangle\rangle\langle\langle 0, \hat{\jmath} \mid-q, p\rangle\rangle),
\end{aligned}
$$

where (2.19) has been used. By (2.21) and (2.25) we obtain

$$
\frac{i}{2} \int_{\Sigma} \omega_{n, m} \wedge \bar{\omega}_{q, p}=\pi\langle\langle n, m \mid-q, p\rangle\rangle=\pi\langle n, m \mid q, p\rangle+i \pi^{2}(p \cdot n-q \cdot m),
$$

so that the monodromy of $f_{n, m}$ corresponds to a surface integral, that is,

$$
f_{n, m}\left(z+\gamma_{p,-q}\right)=e^{\frac{1}{2 \pi i} \int_{\Sigma} \bar{\omega}_{q, p} \wedge \omega_{n, m}} f_{n, m}(z) .
$$

By (2.19), (2.20) and (2.40) the surface integrals $\int_{\Sigma} \omega_{n, m} \wedge \bar{\omega}_{q, p}$ have the properties

$$
\int_{\Sigma} \omega_{n, m} \wedge \bar{\omega}_{q, p}=\int_{\Sigma} \omega_{q, p} \wedge \bar{\omega}_{n, m}+4 \pi^{2}(p \cdot n-q \cdot m)
$$

and

$$
\Im \frac{i}{2} \int_{\Sigma} \omega_{n, m} \wedge \bar{\omega}_{q, p}=\Im \frac{i}{2} \int_{\Sigma} \omega_{m, n} \wedge \bar{\omega}_{p, q} .
$$

The above structure suggests introducing the holomorphic one-differentials

$$
\eta_{j}^{(1)}(z)=\pi \sum_{k=1}^{h}\left(\Omega^{(2)^{-1}}\right)_{j k} \omega_{k}(z), \quad j=1, \ldots, h,
$$

$$
\eta_{j}^{(2)}(z)=\pi \sum_{l=1}^{h}\left[i \delta_{j l}-\sum_{k=1}^{h} \Omega_{j k}^{(1)}\left(\Omega^{(2)^{-1}}\right)_{k l}\right] \omega_{l}(z), \quad j=1, \ldots, h .
$$

By (2.10) and (2.11) it follows that

$$
\omega_{n, m}=\sum_{k=1}^{h}\left(m_{k} \eta_{k}^{(1)}+n_{k} \eta_{k}^{(2)}\right)
$$

moreover

$$
\begin{aligned}
& \Im \oint_{\alpha_{k}} \eta_{j}^{(1)}=0, \quad \Im \oint_{\beta_{k}} \eta_{j}^{(1)}=\pi \delta_{j k}, \\
& \Im \oint_{\alpha_{k}} \eta_{j}^{(2)}=\pi \delta_{j k}, \quad \Im \oint_{\beta_{k}} \eta_{j}^{(2)}=0,
\end{aligned}
$$

$j, k=1, \ldots, h$. Let us set

$$
g_{k}^{(j)}(z) \doteq \exp \int_{z_{0}}^{z} \eta_{k}^{(j)}, \quad j=1,2,
$$

$k=1, \ldots, h$. The expression of $f_{n, m}$ in terms of $g_{k}^{(j)}$ has the simple form

$$
f_{n, m}=\prod_{k=1}^{h} g_{k}^{(1)^{m_{k}}} g_{k}^{(2)^{n_{k}}}
$$


Furthermore, since $\eta_{k}^{(2)}=-\sum_{j=1}^{h} \Omega_{k j} \eta_{j}$, it follows that

$$
f_{n, m}(z)=\prod_{j, k=1}^{h} g_{j}^{(2)}(z)^{D_{k j}^{n m}}=e^{\sum_{j, k=1}^{h} D_{k j}^{n m} \int_{z_{0}}^{z} \eta_{j}},
$$

where the coefficients $D_{k j}^{n m}$ have been defined in (2.15).

\section{EigENFUnCTIONS}

Let $\alpha$ be an element of $\mathcal{K}(\Sigma)$ and let $\omega=\pi i\left(\alpha+i^{\star} \alpha\right)$ be the corresponding holomorphic differential in $\mathcal{H}(\Sigma)$. Let $g$ be the metric on $\Sigma$ given by the line element

$$
d s_{g}=|\omega| .
$$

In local coordinates, if $\omega=h(z) d z$, then

$$
d s_{g}^{2}=|h(z) d z|^{2} .
$$

Thus $g$ defines a flat metric on $\Sigma$ with conical singularities at the $2 h-2$ zeroes of $\omega$. For an account on the geodesic dynamics of such surfaces, see for example [3]. Let $\Delta_{g}$ be the corresponding Laplacian acting on degree zero bundles. In local coordinates,

$$
\Delta_{g}=-|h(z)|^{-2} \partial_{z} \partial_{\bar{z}} .
$$

Theorem 1. If $k \in \mathbb{Z}$ and $F_{k}$ is the single-valued function

$$
F_{k}(z)=e^{2 \pi i k \int_{z_{0}}^{z} \alpha},
$$

then

$$
\Delta_{g} F_{k}=k^{2} F_{k} .
$$

Proof. Immediate.

Let us define the single-valued functions

$$
h_{n, m} \doteq \frac{f_{n, m}}{\bar{f}_{n, m}}=e^{\int^{z} \omega_{n, m}-\overline{\int^{z} \omega_{n, m}}} .
$$

Note that the functions $F_{k}$ correspond to $h_{n, m}^{k}$ for some integer vectors $n$ and $m$. For $k \in \mathbb{Z} \backslash\{0\}$ we have

$$
\int_{\Sigma} \omega_{n, m} \wedge \bar{\omega}_{n, m} \exp k\left(\int^{z} \omega_{n, m}-\overline{\int^{z} \omega_{n, m}}\right)=0,
$$

which follows from the fact that the integrand is a total derivative. Since $\bar{F}_{k}=F_{k}^{-1}$, it follows that the $F_{k}$ satisfy the orthonormality relation

$$
\int_{\Sigma} d \mu F_{k} \bar{F}_{j}=\delta_{j k}
$$

where

$$
d \mu \doteq \frac{\omega \wedge \bar{\omega}}{\int_{\Sigma} \omega \wedge \bar{\omega}}
$$


3.1. Multivaluedness, area and eigenvalues. The area of $\Sigma$ with respect to the metric $d s^{2}=\left|\omega_{n, m}\right|^{2}$ is given by

$$
A_{n, m}=\frac{i}{4} \int_{\Sigma} \omega_{n, m} \wedge \bar{\omega}_{n, m}=\frac{\pi}{2}\langle n, m \mid n, m\rangle=\frac{\pi^{2}}{2}(m-n \cdot \Omega) \cdot \Omega^{(2)}{ }^{-1} \cdot(m-n \cdot \bar{\Omega}) .
$$

The multivaluedness of $f_{n, m}$ is related to $A_{n, m}$. In particular, winding around the cycle $\gamma_{n,-m}=-m \cdot \alpha+n \cdot \beta$, we have

$$
\mathcal{P}_{n,-m} f_{n, m}(z)=e^{-\frac{2 A_{n, m}}{\pi}} f_{n, m}(z)=e^{-\langle n, m \mid n, m\rangle} f_{n, m}(z),
$$

where $\mathcal{P}_{q, p}$ is the winding operator

$$
\mathcal{P}_{q, p} g(z)=g\left(z+\gamma_{p, q}\right) .
$$

Comparing (3.11) with (3.5) we get the following relationship connecting dilatations and eigenvalues:

$$
\lambda_{k}=-\pi \log \frac{\mathcal{P}_{n,-m}^{k^{2}} f_{n, m}(z)}{f_{n, m}(z)} .
$$

Thus we can express the action of the Laplacian on $h_{n, m}^{k}=\left(f_{n, m} / \bar{f}_{n, m}\right)^{k}$ in terms of the winding operator acting on $f_{n, m}$. This relationship between eigenvalues and multivaluedness is reminiscent of a similar relation arising between geodesic lengths (Fuchsian dilatations) and eigenvalues of the Poincaré Laplacian (Selberg trace formula). This is not a surprise. Actually, in the previous sections we have reproduced in the higher genus case some of the structures arising in the case of the torus. In particular, we considered the points $c_{n, m ; k}$ for which the imaginary part of $\sum_{k} c_{n, m ; k} \oint_{\gamma_{p, q}}$ takes values in $\pi \mathbb{Z}$. This is reminiscent of the Poisson summation formula.

3.2. Genus one. Let us denote by $\tau$ the Riemann period matrix in the case of the torus. We set $\tau^{(1)} \doteq \Re \tau$ and $\tau^{(2)} \doteq \Im \tau$. For $h=1$ we have

$$
\omega_{n, m}=c_{n, m} \omega,
$$

with $\omega \doteq \omega_{1}$ the unique holomorphic one-differential on the torus such that $\oint_{\alpha} \omega=$ 1. By (2.11) we have

$$
c_{n, m}=\pi \frac{(m-n \bar{\tau})}{\tau^{(2)}}, \quad(n, m) \in \mathbb{Z}^{2} .
$$

The functions

$$
h_{n, m}=e^{c_{n, m} \int^{z} \omega-\bar{c}_{n, m} \overline{\int^{z} \omega}}, \quad(n, m) \in \mathbb{Z}^{2},
$$

coincide with the well-known eigenfunctions of the Laplacian $\Delta=-2 \partial_{z} \partial_{\bar{z}}$

$$
\Delta h_{n, m}=\lambda_{n, m} h_{n, m}, \quad(n, m) \in \mathbb{Z}^{2},
$$

where

$$
\lambda_{n, m} \doteq 2\left|c_{n, m}\right|^{2}=2 \pi^{2} \frac{(m-n \tau)(m-n \bar{\tau})}{\tau^{(2)^{2}}} .
$$

Under modular transformations of $\tau$ the eigenvalues transform in the following way:

$$
\lambda_{n, m}(\tau+1)=\lambda_{n, m-n}(\tau), \quad \lambda_{n, m}\left(-\frac{1}{\tau}\right)=|\tau|^{2} \lambda_{-m, n}(\tau) .
$$


The results in the previous sections show that the eigenvalues can be generated by winding around the homology cycles, namely

$$
\lambda_{n, m}=2 \pi \frac{\langle n, m \mid n, m\rangle}{\tau^{(2)}}=2 \pi \frac{\Re \oint_{\gamma_{m,-n}} \omega_{n, m}}{\tau^{(2)}} .
$$

In other words, by acting with the winding operator we recover the full spectrum. We now prove modular invariance of $\tau^{(2)^{-1}} \operatorname{det}^{\prime} \Delta$ without computing it (the prime indicates omission of the zero mode of $\Delta$ ). First of all note that

$$
\mu_{n, m}(\tau)=\tau^{(2)} \lambda_{n, m}(\tau), \quad(n, m) \in \mathbb{Z}^{2},
$$

which correspond to the eigenvalues of the Laplacian $\Delta^{\prime}=\tau^{(2)} \Delta$, satisfy

$$
\mu_{\gamma(n, m)}(\gamma \cdot \tau)=\mu_{n, m}(\tau)
$$

where $\gamma(n, m) \doteq(\tilde{n}, \tilde{m})$ with

$$
\left(\begin{array}{c}
\tilde{m} \\
\tilde{n}
\end{array}\right) \doteq\left(\begin{array}{ll}
a & b \\
c & d
\end{array}\right)\left(\begin{array}{c}
m \\
n
\end{array}\right)
$$

and

$$
\gamma \cdot \tau \doteq \frac{a \tau+b}{c \tau+d}, \quad\left(\begin{array}{ll}
a & b \\
c & d
\end{array}\right) \in \operatorname{PSL}(2, \mathbb{Z}) .
$$

By (3.22) we have $\mu_{\gamma(n, m)}(\tau)=\mu_{n, m}\left(\gamma^{-1} \cdot \tau\right)$, that is, any $\mu_{n, m}$, and therefore $\lambda_{n, m}$, can be obtained from a given eigenvalue by modular transformations. The determinants of $\Delta$ and $\Delta^{\prime}$ are related by

$$
\operatorname{det}^{\prime} \Delta^{\prime}=\operatorname{det}^{\prime}\left(\tau^{(2)}\right) \operatorname{det}^{\prime} \Delta=\tau^{(2)}{ }^{-1} \operatorname{det}\left(\tau^{(2)}\right) \operatorname{det}^{\prime} \Delta,
$$

where we used the fact that $c \operatorname{det}^{\prime} c=\operatorname{det} c, c \in \mathbb{C}$. Since $\operatorname{det} \tau^{(2)}$ can be regularized by standard techniques, e.g. by the $\zeta$-function regularization method, to a finite $\tau$-independent constant, we have

$$
\frac{\operatorname{det}^{\prime} \Delta}{\tau^{(2)}}=\mathrm{const} \prod_{(n, m) \in \mathbb{Z}^{2} \backslash\{0,0\}} \mu_{n, m}=\mathrm{const} \prod_{(n, m) \in \mathbb{Z}^{2} \backslash\{0,0\}} \tau^{(2)} \lambda_{n, m} .
$$

Modular invariance of $\tau^{(2)^{-1}} \operatorname{det}^{\prime} \Delta$ now follows by (3.22), namely

$$
\prod_{(n, m) \in \mathbb{Z}^{2} \backslash\{0,0\}} \tau^{(2)} \lambda_{n, m}(\tau)=\prod_{\gamma \in P S L(2, \mathbb{Z})} \mu_{N, M}(\gamma \cdot \tau),
$$

where $N, M$ are arbitrary integers not simultaneously vanishing. It follows that $\operatorname{det}^{\prime} \Delta$ can be expressed as the product of all modular transformations acting on an arbitrary eigenvalue

$$
\operatorname{det}^{\prime} \Delta=\operatorname{const} \tau^{(2)} \prod_{\gamma \in P S L(2, \mathbb{Z})} \mu_{N, M}(\gamma \cdot \tau)
$$

Note that modular invariance of $\tau^{(2)^{-1}} \operatorname{det}^{\prime} \Delta$ essentially implies that $\tau^{(2)^{-1}} \operatorname{det}^{\prime} \Delta$ $=\tau^{(2)}|\eta(\tau)|^{4}$, where $\eta(\tau)$ is the Dedekind $\eta$-function. 


\section{Special Riemann surfaces}

In general there are other eigenfunctions besides $h_{n, m}^{k}, k \in \mathbb{N}$. For example, when all the $2 m_{j}$ 's and $2 n_{j}$ 's are integer multiple of an integer $N$, then the eigenfunctions include $h_{n, m}^{k}, k \in \mathbb{N}$ whose eigenvalue is $2 A_{n, m} k^{2} / N^{2}$.

More generally one should investigate whether the period matrix has some nontrivial number theoretical structure. To see this we first note the trivial fact that since for $h=1$ the space of holomorphic one-differentials is one-dimensional, it follows that the ratio between $\omega_{n, m}$ and $\omega_{n^{\prime}, m^{\prime}}$ is always a constant. This allows one to construct the infinite set of eigenvalues labelled by two integers $(n, m) \in \mathbb{Z}^{2}$. In the case $h \geq 2$ the ratio $\omega_{n, m} / \omega_{n^{\prime}, m^{\prime}}$ is in general not a constant. This is the reason why we considered the eigenfunctions of the kind $h_{n, m}^{k}$ with fixed $(n, m) \in \mathbb{Z}^{2 h}$. However there are other interesting possibilities. For example, if, besides $\alpha,{ }^{\star} \alpha$ is also integral, then for $k_{1}, k_{2} \in \mathbb{Z}$, the single valued function

$$
F_{k_{1}, k_{2}}=e^{2 \pi i \int_{z_{0}}^{z}\left(k_{1} \alpha+k_{2}{ }^{\star} \alpha\right)}
$$

satisfies

$$
\Delta_{g} F_{k_{1}, k_{2}}=\left(k_{1}^{2}+k_{2}^{2}\right) F_{k_{1}, k_{2}} .
$$

Note that since $k_{1} \alpha+k_{2}{ }^{\star} \alpha=2 i \Im\left[\left(k_{1}-i k_{2}\right) \omega\right]$, it follows that integrality of both $\alpha$ and ${ }^{\star} \alpha$ is a particular case of a more general one.

Theorem 2. If the holomorphic one-differentials $\omega_{n, m}$ and $\omega_{n^{\prime}, m^{\prime}}$ satisfy the equation

$$
\omega_{n^{\prime}, m^{\prime}}(z)=c \omega_{n, m}(z)
$$

with both $(n, m)$ and $\left(n^{\prime}, m^{\prime}\right)$ in $\mathbb{Z}^{2 h}$ and $c \in \mathbb{C} \backslash \mathbb{Q}$, then the single valued function

$$
h_{n^{\prime}, m^{\prime}}=e^{c \int^{z} \omega_{n, m}-\bar{c} \overline{\int^{z} \omega_{n, m}}} \neq h_{n, m}^{k} \quad c \in \mathbb{C} \backslash \mathbb{Q}, \quad k \in \mathbb{Q},
$$

satisfies

$$
\Delta_{g^{n, m}} h_{n^{\prime}, m^{\prime}}=\lambda_{c} h_{n^{\prime}, m^{\prime}}
$$

where

$$
\lambda_{c}=2 A_{n, m}|c|^{2}
$$

and

$$
c=\frac{m_{i}^{\prime}-\sum_{k=1}^{h} \bar{\Omega}_{i k} n_{k}^{\prime}}{m_{i}-\sum_{k=1}^{h} \bar{\Omega}_{i k} n_{k}}=\frac{m_{j}^{\prime}-\sum_{k=1}^{h} \bar{\Omega}_{j k} n_{k}^{\prime}}{m_{j}-\sum_{k=1}^{h} \bar{\Omega}_{j k} n_{k}}, \quad i, j=1, \ldots, h .
$$

Proof. It is immediate to check (4.5). The only point is to find the expression of $c$. This follows by the observation that since the holomorphic one-differentials $\omega_{1}, \ldots, \omega_{h}$ are linearly independent, 4.3) is equivalent to

$$
m_{j}^{\prime}-\sum_{k=1}^{h} \Omega_{j k} n_{k}^{\prime}=\bar{c}\left(m_{j}-\sum_{k=1}^{h} \Omega_{j k} n_{k}\right), \quad j=1, \ldots, h .
$$

Note that to each $(n, m)$ and $\left(n^{\prime}, m^{\prime}\right)$ satisfying (4.8) there is a possible value of $c \equiv c\left(n, m ; n^{\prime}, m^{\prime}\right)$.

Thus we can reproduce in higher genus the basic structure (3.16)-(3.18) considered in the torus case and then find eigenvalues with a nontrivial dependence on the complex structure. 
The problem of finding the possible (in general complex) solutions of (4.3) is strictly related to the number theoretical properties of $\Omega$.

Observe that

$$
\Delta_{g^{n^{\prime}, m^{\prime}}} h_{n, m}=\lambda_{c}{ }^{\prime} h_{n, m},
$$

where

$$
\lambda_{c}{ }^{\prime}=4 \frac{A_{n, m} A_{n^{\prime}, m^{\prime}}}{\lambda_{c}} .
$$

We will call special the Riemann surfaces admitting solutions of (4.8) with nonrational values of $c$. Since a change in the sign of $c$ is equivalent to a change sign of either $(m, n)$ or $\left(m^{\prime}, n^{\prime}\right)$, in the following, without loss of generality, we will assume that

$$
\Im \bar{c}>0 .
$$

4.1. The solution space. Equation (4.8) has solutions if there are integers $(n, m)$ and $\left(n^{\prime}, m^{\prime}\right)$ such that the period matrix satisfies the $h-1$ consistency conditions

$$
\frac{m_{i}^{\prime}-\sum_{k=1}^{h} \Omega_{i k} n_{k}^{\prime}}{m_{i}-\sum_{k=1}^{h} \Omega_{i k} n_{k}}=\frac{m_{j}^{\prime}-\sum_{k=1}^{h} \Omega_{j k} n_{k}^{\prime}}{m_{j}-\sum_{k=1}^{h} \Omega_{j k} n_{k}}, \quad i, j=1, \ldots, h .
$$

Thus, for $(n, m) \in \mathbb{Z}^{2 h}$ fixed, the Laplacian $\Delta_{g^{n, m}}$ has eigenvalues

$$
\lambda_{c}=2 A_{n, m}\left|\frac{m_{i}^{\prime}-\sum_{k=1}^{h} \bar{\Omega}_{i k} n_{k}^{\prime}}{m_{i}-\sum_{k=1}^{h} \bar{\Omega}_{i k} n_{k}}\right|^{2}, \quad\left(n^{\prime}, m^{\prime}\right) \in \mathcal{S}_{n, m}(\Omega),
$$

where $\mathcal{S}_{n, m}(\Omega)$ denotes the solution space

$$
\mathcal{S}_{n, m}(\Omega) \doteq\left\{\left(n^{\prime}, m^{\prime}\right) \mid \omega_{n^{\prime}, m^{\prime}}=c \omega_{n, m},\left(n^{\prime}, m^{\prime}\right) \in \mathbb{Z}^{2 h}\right\} .
$$

For a given $\Omega$ the space $\mathcal{S}_{n, m}(\Omega)$ may contain other points besides $(k n, k m), k \in \mathbb{Z}$. To investigate the structure of such a space we should understand the nature of the Riemann surfaces whose period matrix satisfies (4.8). To this end we note that (4.8) has been suggested in order to reproduce in higher genus the basic structure (3.16)-(3.18) considered in the torus case. So, we should expect a Riemann surface strictly related to the torus geometry. Remarkably, this is in fact the case, as we have the following

Theorem 3. The Riemann surfaces with period matrices satisfying (4.8) correspond to branched covering of the torus.

Proof. First of all note that by (4.8) it follows that the function

$$
w(z)=\int^{z} \hat{\omega}
$$

where $\hat{\omega} \doteq\left(\bar{c} n-n^{\prime}\right) \cdot \omega$, has monodromy

$$
\oint_{\gamma_{p, q}} \hat{\omega}=-p \cdot n^{\prime}-q \cdot m^{\prime}+\bar{c}(p \cdot n+q \cdot m),
$$

implying that $w$ is a holomorphic map from $\Sigma$ to the torus with period matrix $\bar{c}$. Vice versa, if $w$ is a holomorphic map of a branched covering of the torus to the 
torus itself, then $w\left(z+\gamma_{p, q}\right)=w(z)+p \cdot N^{\prime}+q \cdot M^{\prime}+\tau(p \cdot N+q \cdot M)$, for some integer vectors $M, N, M^{\prime}, N^{\prime}$, and by the Riemann bilinear relations

$$
0=\int_{\Sigma} \omega_{k} \wedge d w=M_{k}^{\prime}+\tau M_{k}-\sum_{j=1}^{h}\left(N_{j}^{\prime}+\tau N_{j}\right) \Omega_{j k}, \quad k=1, \ldots, h
$$

which is (4.8) with $\tau=\bar{c}$ (see [4] for explicit constructions of branched covering of the torusi $)$.

Remark 1. Equation (1.2), derived in 1] (see (5.18) and (5.23) there), has been subsequently and independently derived in [5] by studying the null compactification of type-IIA-string perturbation theory at finite temperature. In [5] an equivalent proof of Theorem 3 is also provided.

4.2. Metric induced by the covering. By means of the map $w$ from $\Sigma$ to the torus with period matrix $\operatorname{sign}(\Im(\bar{c})) \bar{c}$ we can construct an infinite set of eigenfunctions for the Laplacian $\Delta=-2|\hat{\omega}|^{-2} \partial_{z} \partial_{\bar{z}}$ on $\Sigma$ defined with respect to the metric $|\hat{\omega}|^{2}$. These eigenfunctions are

$$
h_{n, m}=e^{c_{n, m} \int^{z} \hat{\omega}-\bar{c}_{n, m} \int^{z} \hat{\omega}}, \quad(n, m) \in \mathbb{Z}^{2},
$$

corresponding to the eigenvalues

$$
\lambda_{n, m}=2\left|c_{n, m}\right|^{2}=2 \pi^{2} \frac{(m-c n)(m-\bar{c} n)}{c^{(2)^{2}}},
$$

where

$$
c_{n, m}=\pi \frac{(m-n \bar{c})}{c^{(2)}}, \quad(n, m) \in \mathbb{Z}^{2} .
$$

The proof is a direct consequence of the relation $\Delta=-2|\hat{\omega}|^{-2} \partial_{z} \partial_{\bar{z}}=-2 \partial_{w} \partial_{\bar{w}}$.

Let us set

$$
D_{j} \doteq \sum_{k=1}^{h} \bar{D}_{k j}^{m n}, \quad D_{j}^{\prime} \doteq \sum_{k=1}^{h} \bar{D}_{k j}^{m^{\prime} n^{\prime}} .
$$

By (4.7) and the Poisson summation formula, we have

$$
\sum_{n=-\infty}^{\infty} e^{-n^{2} \pi \frac{D_{j}^{\prime}}{D_{j}}}=\sqrt{\frac{D_{j}}{D_{j}^{\prime}}} \sum_{n=-\infty}^{\infty} e^{-n^{2} \pi \frac{D_{j}}{D_{j}^{\prime}}}, \quad j=1, \ldots, h .
$$

4.3. Genus 2. Before considering (4.12) for arbitrary genus, it is instructive to investigate the $h=2$ case. Since $\Omega_{i j}=\Omega_{j i}$ we have

$$
\frac{m_{1}^{\prime}-\Omega_{11} n_{1}^{\prime}-\Omega_{12} n_{2}^{\prime}}{m_{1}-\Omega_{11} n_{1}-\Omega_{12} n_{2}}=\frac{m_{2}^{\prime}-\Omega_{12} n_{1}^{\prime}-\Omega_{22} n_{2}^{\prime}}{m_{2}-\Omega_{12} n_{1}-\Omega_{22} n_{2}} .
$$

Thus the problem is the following: given $\Omega_{11}$ and $\Omega_{12}$ find all the integers $(n, m$; $\left.n^{\prime}, m^{\prime}\right) \in \mathbb{Z}^{8}$ such that (4.23) is satisfied. The solution of this problem depends on structure of $\Omega$.

We consider period matrices satisfying the relation

$$
\Omega_{22}=\frac{\hat{N}_{1}}{\hat{N}_{4}} \Omega_{11}+\frac{\hat{N}_{2}}{\hat{N}_{4}} \Omega_{12}+\frac{\hat{N}_{3}}{\hat{N}_{4}}, \quad\left(\hat{N}_{1}, \hat{N}_{2}, \hat{N}_{3}, \hat{N}_{4}\right) \in \mathbb{Z}^{4}, \quad \hat{N}_{4} \neq 0,
$$

\footnotetext{
${ }^{1}$ I am grateful to the anonymous referee for suggesting [4].
} 
that is,

$$
\Omega_{i j}=\left(\begin{array}{cc}
\Omega_{11} & \Omega_{12} \\
\Omega_{12} & N_{1} \Omega_{11}+N_{2} \Omega_{12}+N_{3}
\end{array}\right),
$$

where $N_{i} \doteq \hat{N}_{i} / \hat{N}_{4}, i=1,2,3$. Positivity of $\Omega_{i j}^{(2)}$ implies the following condition on $N_{1}, N_{2}, N_{3}$ :

$$
\Omega_{11}^{(2)}\left(N_{1} \Omega_{11}^{(2)}+N_{2} \Omega_{12}^{(2)}+N_{3}\right)>\Omega_{12}^{(2)^{2}} .
$$

With the position (4.24), equation (4.23) becomes

$$
\frac{m_{1}^{\prime}-\Omega_{11} n_{1}^{\prime}-\Omega_{12} n_{2}^{\prime}}{m_{1}-\Omega_{11} n_{1}-\Omega_{12} n_{2}}=\frac{m_{2}^{\prime}-N_{3} n_{2}^{\prime}-\Omega_{11} N_{1} n_{2}^{\prime}-\Omega_{12}\left(n_{1}^{\prime}+N_{2} n_{2}^{\prime}\right)}{m_{2}-N_{3} n_{2}-\Omega_{11} N_{1} n_{2}-\Omega_{12}\left(n_{1}+N_{2} n_{2}\right)} .
$$

We will look for solutions of this equation of the form

$$
\begin{aligned}
& m_{2}-N_{3} n_{2}-\Omega_{11} N_{1} n_{2}-\Omega_{12}\left(n_{1}+N_{2} n_{2}\right)=N\left[m_{1}-\Omega_{11} n_{1}-\Omega_{12} n_{2}\right], \\
& m_{2}^{\prime}-N_{3} n_{2}^{\prime}-\Omega_{11} N_{1} n_{2}^{\prime}-\Omega_{12}\left(n_{1}^{\prime}+N_{2} n_{2}^{\prime}\right)=N\left[m_{1}^{\prime}-\Omega_{11} n_{1}^{\prime}-\Omega_{12} n_{2}^{\prime}\right],
\end{aligned}
$$

with $N \in \mathbb{Q}$. Since $\left(n, m ; n^{\prime}, m^{\prime}\right) \in \mathbb{Z}^{8}$, the general solutions not depending on $\Omega_{11}$ and $\Omega_{12}$ are

$$
\begin{array}{lll}
m_{2}-N_{3} n_{2}=N m_{1}, & n_{1}+N_{2} n_{2}=N n_{2}, & N_{1} n_{2}=N n_{1}, \\
m_{2}^{\prime}-N_{3} n_{2}^{\prime}=N m_{1}^{\prime}, & n_{1}^{\prime}+N_{2} n_{2}^{\prime}=N n_{2}^{\prime}, & N_{1} n_{2}^{\prime}=N n_{1}^{\prime} .
\end{array}
$$

Note that each solution of (4.30) defines a metric $g^{n, m}$ whereas the solutions of (4.31) give, by (4.13), the eigenvalues $\lambda_{c}$ of $\Delta_{g^{n, m}}$.

The compatibility condition for (4.31) constrains $N$ to be

$$
N_{ \pm}=\frac{N_{2} \pm \sqrt{N_{2}^{2}+4 N_{1}}}{2} .
$$

Since $N \in \mathbb{Q}$ and $N_{i} \in \mathbb{Z} / \hat{N}_{4}, i=1,2,3$, we have

$$
N_{1}=M N_{2}+M^{2}, \quad M \in\left\{k \in \mathbb{Q} \mid\left(k N_{2}+k^{2}\right) \in \mathbb{Z} / \hat{N}_{4}\right\} .
$$

Equation (4.30) has a double set of solutions. In the case $N=N_{+}=N_{2}+M$, we have

$$
n_{2}=\frac{n_{1}}{M}, \quad m_{2}=\left(N_{2}+M\right) m_{1}+\frac{N_{3} n_{1}}{M}, \quad\left(n_{1}, m_{1}\right) \in \Gamma^{(+)},
$$

where

$$
\Gamma^{(+)} \doteq\left\{(k, j) \in \mathbb{Z}^{2} \mid\left(\frac{k}{M},\left(N_{2}+M\right) j+\frac{N_{3} k}{M}\right) \in \mathbb{Z}^{2}\right\} .
$$

In the second case $N=N_{-}=-M$, so that

$$
n_{2}=-\frac{n_{1}}{N_{2}+M}, \quad m_{2}=-M m_{1}-\frac{N_{3} n_{1}}{N_{2}+M}, \quad\left(n_{1}, m_{1}\right) \in \Gamma^{(-)},
$$

where

$$
\Gamma^{(-)} \doteq\left\{(k, j) \in \mathbb{Z}^{2} \mid\left(\frac{k}{N_{2}+M}, M j+\frac{N_{3} k}{N_{2}+M}\right) \in \mathbb{Z}^{2}\right\} .
$$

Note that given $N_{1}, N_{2}$ and $N_{3}$ we found that $N_{1}=M N_{2}+M^{2}$ and either $N=$ $N_{2}+M$ or $-M$. Therefore it is natural to choose $M, N_{2}$ and $N_{3}$ to parametrize 
$\Omega_{22}$. Note also that by (4.24) and (4.33) it follows that $M$ and $-N_{2}-M$ correspond to the same value of $\Omega_{22}$.

Given $M, N_{2}, N_{3}, N_{4}$ there are two sets of Laplacians parametrized by points in $\Gamma^{( \pm)}$. Let $\left(n_{1}, m_{1}\right)$ be a point in $\Gamma^{(+)}$and $\left(n_{2}, m_{2}\right)$ given by (4.34). The first set of eigenvalues is

$$
\lambda_{c}^{(+)}=2 A_{n, m} \frac{\left|M m_{1}^{\prime}-n_{1}^{\prime}\left(M \Omega_{11}+\Omega_{12}\right)\right|^{2}}{\left|M m_{1}-n_{1}\left(M \Omega_{11}+\Omega_{12}\right)\right|^{2}},
$$

where

$$
n_{2}^{\prime}=\frac{n_{1}^{\prime}}{M}, \quad m_{2}^{\prime}=\left(N_{2}+M\right) m_{1}^{\prime}+\frac{N_{3} n_{1}^{\prime}}{M}, \quad\left(n_{1}^{\prime}, m_{1}^{\prime}\right) \in \Gamma^{(+)} .
$$

Let $\left(n_{1}, m_{1}\right)$ be a point in $\Gamma^{(-)}$and $\left(n_{2}, m_{2}\right)$ given by (4.36). The second set of eigenvalues is

$$
\lambda_{c}^{(-)}=2 A_{n, m} \frac{\left|\left(N_{2}+M\right) m_{1}^{\prime}-n_{1}^{\prime}\left[\left(N_{2}+M\right) \Omega_{11}-\Omega_{12}\right]\right|^{2}}{\left|\left(N_{2}+M\right) m_{1}-n_{1}\left[\left(N_{2}+M\right) \Omega_{11}-\Omega_{12}\right]\right|^{2}},
$$

where

$$
n_{2}^{\prime}=-\frac{n_{1}^{\prime}}{N_{2}+M}, \quad m_{2}^{\prime}=-M m_{1}-\frac{N_{3} n_{1}}{N_{2}+M}, \quad\left(n_{1}^{\prime}, m_{1}^{\prime}\right) \in \Gamma^{(-)} .
$$

These eigenvalues have a structure which is similar to the structure of the ones of the Laplacian on the torus.

4.4. Higher genus. We now consider the higher genus case. By (4.12) we have

$$
\begin{aligned}
& m_{i}-\sum_{k=1}^{h} \Omega_{i k} n_{k}=N_{i j}\left(m_{j}-\sum_{k=1}^{h} \Omega_{j k} n_{k}\right), \\
& m_{i}^{\prime}-\sum_{k=1}^{h} \Omega_{i k} n_{k}^{\prime}=N_{i j}\left(m_{j}^{\prime}-\sum_{k=1}^{h} \Omega_{j k} n_{k}^{\prime}\right),
\end{aligned}
$$

$i, j=1, \ldots, h$. Equation (4.42) gives the constraint on the structure of the metric $g^{n, m}$. Note that

$$
N_{i j} N_{j k}=N_{i k}, \quad i, j=1, \ldots, h,
$$

in particular, $N_{i j}=N_{j i}^{-1}$. The matrix $N_{i j}$ is determined by $h-1$ elements. For example, since $N_{i j}=N_{i 1} N_{1 j}=N_{1 i}^{-1} N_{1 j}$, in terms of $N_{12}, \ldots, N_{1 h}$ we have

$$
N_{i j}=\left(\begin{array}{ccccc}
1 & N_{12} & N_{13} & \ldots & N_{1 h} \\
N_{12}^{-1} & 1 & N_{13} N_{12}^{-1} & \ldots & N_{1 h} N_{12}^{-1} \\
\vdots & \vdots & \vdots & \vdots & \vdots \\
N_{1 h}^{-1} & N_{1 h}^{-1} N_{12} & N_{1 h}^{-1} N_{13} & \ldots & 1
\end{array}\right)
$$

which has vanishing determinant.

Since $\Omega$ is symmetric, it follows that in each one of the $h-1$ equations (4.42) (or (4.43) ) there is always one, and only one, matrix element appearing in both sides. In other words, both sides of (4.42) contain $\Omega_{i j}=\Omega_{j i}$. Therefore, it is natural to consider period matrices of the form

$$
\Omega_{i j}=\sum_{k, l=1}^{h} N_{i j}^{k l} \Omega_{k l}+M_{i j}, \quad N_{i j}^{k l} \in \mathbb{Q}, \quad M_{i j} \in \mathbb{Q}, \quad N_{i j}^{i j}=0,
$$


and then, for each pair $i, j$, to substitute it in the equation involving $N_{i j}$. This allows us to transform, for each $i, j$, (4.42) and (4.43) in equations containing all the matrices elements but $\Omega_{i j}$ on both sides. Note that the symmetry of $\Omega_{i j}$ implies that $N_{i j}^{k l}$ is symmetric in $i, j$ and $k, l$ separately and $M_{i j}=M_{j i}$. By (4.46) we have that (4.42) and (4.43) become

$$
\begin{gathered}
N_{i j} m_{j}-m_{i}+\sum_{k, l=1}^{h}\left\{\left(n_{j}-n_{i} N_{i j}\right)\left(N_{i j}^{k l} \Omega_{k l}+M_{i j}\right)\right. \\
\left.+\Omega_{k l}\left[\delta_{i k}\left(n_{l}-\delta_{l j} n_{j}\right)-\delta_{j k}\left(n_{l}-\delta_{l i} n_{i}\right) N_{i j}\right]\right\}=0, \\
N_{i j} m_{j}^{\prime}-m_{i}^{\prime}+\sum_{k, l=1}^{h}\left\{\left(n_{j}^{\prime}-n_{i}^{\prime} N_{i j}\right)\left(N_{i j}^{k l} \Omega_{k l}+M_{i j}\right)\right. \\
\left.+\Omega_{k l}\left[\delta_{i k}\left(n_{l}^{\prime}-\delta_{l j} n_{j}^{\prime}\right)-\delta_{j k}\left(n_{l}^{\prime}-\delta_{l i} n_{i}^{\prime}\right) N_{i j}\right]\right\}=0,
\end{gathered}
$$

$i, j=1, \ldots, h$. We do not investigate the conditions following from (4.42) and (4.43) further, rather we shortly consider the period matrices satisfying the conditions

$$
\Omega_{i k}=\sum_{l=1}^{h} N_{i k, j}^{l} \Omega_{j l}+M_{i k}, \quad N_{i k, j}^{l} \in \mathbb{Q}, M_{i k} \in \mathbb{Q}, \quad i, j, k=1, \ldots, h .
$$

Substituting $\Omega_{i k}$ in the left-hand side of (4.42) and (4.43) these transform in simplified equations, as now they involve matrix elements with the same value of the first index.

Substituting $\Omega_{j l}$ in the RHS of (4.49) with $\sum_{m=1}^{h} N_{j l, n}^{m} \Omega_{n m}+M_{j l}$, we have

$$
\Omega_{i k}=\sum_{l=1}^{h} N_{i k, j}^{l}\left(\sum_{m=1}^{h} N_{j l, n}^{m} \Omega_{n m}+M_{j l}\right)+M_{i k}, \quad i, j, k, n=1, \ldots, h .
$$

Comparing (4.49) with (4.50) one obtains a set of equations that, once one makes the additional requirement that the terms involving the period matrix cancel separately, become

$$
\begin{gathered}
\sum_{l=1}^{h} N_{i k, j}^{l} N_{j l, n}^{m}=N_{i k, n}^{m}, \quad i, j, k, m, n=1, \ldots, h, \\
\sum_{l=1}^{h} N_{i k, j}^{l} M_{j l}=0, \quad i, j, k=1, \ldots, h .
\end{gathered}
$$

4.5. Special Riemann surfaces and complex multiplication. We now show that special Riemann surfaces have a Jacobian with complex multiplication $(\mathrm{CM})$ First note that in terms of

$$
v_{k} \doteq m_{k}-\sum_{j=1}^{h} \Omega_{k j} n_{j}, \quad v_{k}^{\prime} \doteq m_{k}^{\prime}-\sum_{j=1}^{h} \Omega_{k j} n_{j}^{\prime},
$$

\footnotetext{
${ }^{2}$ Jacobian with CM have been recently considered in the framework of rational CFT [ 6 .
} 
(4.12) reads

$$
v_{j} v_{k}^{\prime}-v_{k} v_{j}^{\prime}=0, \quad \forall j, k,
$$

which is equivalent to

$$
\Omega N \Omega+\Omega M-\tilde{M} \Omega-M^{\prime}=0,
$$

where $\sim$ denotes the transpose and

$$
N_{j k} \doteq n_{j}^{\prime} n_{k}-n_{k}^{\prime} n_{j}, \quad M_{j k} \doteq m_{j}^{\prime} n_{k}-m_{k} n^{\prime} j, \quad M_{j k}^{\prime} \doteq m_{j}^{\prime} m_{k}-m_{k}^{\prime} m_{j} .
$$

On the other hand, a Jacobian is said to admit complex multiplication if there exist integer matrices $N, M, M^{\prime}$ and $N^{\prime}$ such that

$$
\Omega(M+N \Omega)=M^{\prime}+N^{\prime} \Omega,
$$

that is,

$$
\Omega N \Omega+\Omega M-N^{\prime} \Omega-M^{\prime}=0 .
$$

Comparing with 4.55 , we see that the Jacobians of special Riemann surfaces admit a particular kind of CM. According to Theorem 3 this CM is the one of a Jacobian corresponding to a branched covering of the torus.

\section{REFERENCES}

[1] M. Matone, Uniformization theory and 2D gravity I. Liouville action and intersection numbers, Int. J. Mod. Phys. A10 (1995) 289 - 336.

[2] H.M. Farkas and I. Kra, Riemann Surfaces, Springer-Verlag (1992). MR 93a:30047

[3] Y.M. Vorobets, Ergodicity of billiards in polygons: explicit examples, Russ. Math. Surv. 51 (1996) $779-817$. MR 97j:58092

[4] P.J. Richens and M.V. Berry, Pseudointegrable systems in classical and quantum mechanics, Physica D 2 (1981) 495 - 512. MR 82k:58050

[5] G. Grignani, P. Orland, L. D. Paniak and G. W. Semenoff, Matrix theory interpretation of DLCQ string worldsheets, Phys. Rev. Lett. 85 (2000) 3343 - 3346.

[6] S. Gukov and C. Vafa, Rational Conformal Field Theories and Complex Multiplication, hepth/0203213.

Department of Physics "G. Galilei" - Istituto Nazionale di Fisica Nucleare, UniverSity of Padova, Via Marzolo, 8 - 35131 Padova, Italy

E-mail address: matone@pd.infn.it 